\title{
ON MEASURABILITY AND REGULARITY
}

W. JOHN WILBUR

1. Introduction. In its most abstract form the fundamental question concerning nonmeasurability is the question of the existence of measurable cardinals (see $[7$, p. 307]). If a cardinal $\aleph$ is not measurable then a nontrivial totally finite measure cannot be defined on the power set of $\boldsymbol{\aleph}$. Thus for such a measure defined on subsets of $\boldsymbol{\aleph}$ we are assured of the existence of nonmeasurable sets. One reason why it might be desirable to take a less abstract approach to nonmeasurability is the following. While the existence of measurable cardinals has come to appear quite plausible (see $[1$, pp. 80,81$]$ and $[7, \mathrm{p}$. $307]$ ), for a more restricted class of measures it may yet always be possible to find nonmeasurable sets regardless of the cardinality of the underlying space. For example in $[4$, pp. 226, 227] it is shown that every nondiscrete locally compact group contains a subset that is nonmeasurable under every left translation invariant extension of left Haar measure.

In this paper, assuming the axiom of choice, we shall demonstrate the existence of nonmeasurable sets for any nontrivial regular measure defined on a locally compact Hausdorff space. In the case when the space is a metric space this problem has been solved (see $[5, \mathrm{p}$. $146]$ or $[4$, pp. 218,219$])$, but the methods do not seem amenable to direct generalization. We shall obtain our result by constructing a suitable continuous function from the space in question to the unit interval.

2. Preliminaries. Throughout, $P$ will be a locally compact Hausdorff topological space. The families of compact and open subsets of $P$ will be denoted by $\mathcal{C}$ and $Q$, respectively. The $\sigma$-algebra generated by $Q$, i.e., the Borel sets, will be denoted by $ß$. If $(P, \mathfrak{T}, \mu)$ is a measure space, $\mu$ will be called regular iff:

(i) $B \subset \mathfrak{T}$;

(ii) $\mu(C)<\infty$, for all $C \in \mathfrak{e}$;

(iii) $\mu(O)=\sup \{\mu(C) \mid C \subset O, C \in \mathbb{e}\}$ for all $O \in a$;

(iv) $\mu(A)=\inf \{\mu(O) \mid A \subset O, O \in Q\}$ for all $A \in \mathfrak{T}$.

This definition of regularity is taken from [5, p. 177]. It reduces to that given in [3, p. 224] when $P$ is $\sigma$-compact and $B=\mathfrak{T}$. If $\mu$ is regular, $A \in \mathfrak{T}$, and $\mu(A \cap C)=0$ for every $C \in \mathcal{C}$ we shall call $A$ locally $\mu$-null (see $[\mathbf{5}$, p. 122]).

\footnotetext{
Received by the editors March 29, 1968.
} 
If $(X, \mathfrak{T}, \mu)$ is a measure space, a set $A \in \mathscr{T}$ will be called an atom iff $\mu(A)>0$ and for every $B \in \mathfrak{N}$ with $B \subset A, \mu(B)=0$ or $\mu(A-B)=0$. If $A$ and $B$ are members of $\mathfrak{T}$ we shall call $A$ and $B$ equivalent iff $\mu((A-B) \cup(B-A))=0$. Two atoms $A$ and $B$ are plainly equivalent iff $\mu(A \cap B)>0$.

For an arbitrary subset $Q$ of $P$ we shall let $\partial Q$ stand for the topological boundary of $Q$ in $P$ and $Q^{-}$stand for the closure of $Q$ in $P$.

3. The results. If $(P, \mathfrak{T}, \mu)$ is a measure space and $\mu$ a regular measure any singleton of positive measure must be an atom. Our first result indicates that, except possibly for locally $\mu$-null sets, the atoms of a regular measure must be equivalent to singleton atoms.

3.1. TheOREM. Let $(P, \mathfrak{T}, \mu)$ be a measure space where $\mu$ is regular. Then every atom is either locally $\mu$-null or equivalent to a singleton atom.

Proof. Let $A$ be an atom and suppose $A$ is not locally $\mu$-null. Then there is a $C \in \mathcal{C}$ with $\mu(A \cap C)>0$ and hence $A$ and $A \cap C$ must be equivalent atoms. Thus it will be sufficient to show that $A \cap C$ is equivalent to some singleton atom. Now $A \cap C$ will be equivalent to a singleton which is an atom if and only if there is an $x \in A \cap C$ with $\mu(\{x\})>0$. We shall show that there is such an $x$. Let $\mathcal{F}$ be the family of compact subsets of $A \cap C$ with positive measure. It is a consequence of the regularity of $\mu$ and the finiteness of $\mu(A \cap C)$ that $\mathfrak{F}$ is nonempty. Since $\mathcal{F}$ is a family of equivalent atoms it also has the finite intersection property. We conclude that $\cap_{F}$ is a nonempty compact set. The proof will be completed by showing that $\cap \mathcal{F}$ is a singleton and $\mu(\cap \mathcal{F})>0$. Suppose $x, y \in \cap \mathcal{F}$ with $x \neq y$. Let $C^{\prime} \in \mathcal{F}$ and choose an open neighborhood $O$ of $x$ such that $y \notin O$. Since $C^{\prime}$ is an atom either $\mu\left(C^{\prime}-O\right)=0$ or $\mu\left(C^{\prime} \cap O\right)=0$. If $\mu\left(C^{\prime}-O\right)=0$ then $\mu\left(C^{\prime} \cap O\right)>0$ and by the regularity of $\mu$ there must be a $C^{\prime \prime} \in \mathcal{F}$ with $C^{\prime \prime} \subset C^{\prime} \cap O$. In this case $y \notin \cap F$, a contradiction. On the other hand if $\mu\left(C^{\prime} \cap O\right)=0$ then $\mu\left(C^{\prime}-O\right)>0$ and $C^{\prime}-O \in \mathcal{F}$ and $x \notin \cap F$, a contradiction. It follows that $\cap F$ is a singleton. To see that $\mu(\cap \mathcal{F})>0$, let $O \in Q$ with $\cap F \subset O$. Then there is a finite subfamily $\mathcal{E}$ of $\mathfrak{F}$ with $\cap \mathcal{E} \subset 0$. But we must also have $\cap \mathcal{E} \in \mathcal{F}$. Thus $\mu(O) \geqq \mu(\cap \mathcal{E})=\mu(A \cap C)>0$. Employing the regularity of $\mu$, we have

$$
\mu(\cap F)=\inf \{\mu(O) \mid \cap F \subset O, O \in Q\} \geqq \mu(A \cap C)>0 .
$$

This result is of independent interest. It is an open question whether a locally $\mu$-null set can be an atom. If the space is paracompact, however, this question is easily settled in the negative by applying a result given in $[2$, p. 241] which indicates that a locally 
compact paracompact space is a disjoint union of open $\sigma$-compact subspaces.

In order to apply Theorem 3.1 to our problem we shall need the following lemma which appears as an exercise in [3, p. 174]. The reader will find no difficulty in constructing a proof by the method of exhaustion.

3.2. Lemma. Let $(X, \mathfrak{T}, \mu)$ be a measure space which has no atoms. Let $A \in \mathbb{M}$ with $\mu(A)<\infty$. Then if $0 \leqq r \leqq 1$ there is a set $B \in \mathbb{M}$ with $\mu(B)=r \mu(A)$.

Let $\left\{\left(P, \mathfrak{T H}_{i}, \mu_{i}\right)\right\}_{i=1}^{\infty}$ be a family of measure spaces with each $\mu_{i}$ regular. We shall let $\odot$ stand for the family of all open sets $O$ such that $\mu_{i}(\partial O)=0, i=1,2, \cdots$.

\subsection{Lemma. The family $\odot$ is closed under finite unions.}

Proof. This follows immediately from the fact that if $\left\{O_{i}\right\}_{i=1}^{n}$ is a finite subfamily of $\rho$ then $\partial\left(\bigcup_{i=1}^{n} O_{i}\right) \subset \bigcup_{i=1}^{n} \partial O_{i}$.

\subsection{Lemma. The family $\odot$ is a base for the topology of $P$.}

Proof. Let $x \in P$ and let $O \in Q$ with $x \in O$. We must find an open subset $V$ with $x \in V \subset O$ and $\mu_{i}(\partial V)=0$ for each $i$. By local compactness we may choose a compact neighborhood $C$ of $x$ with $C \subset O$. If $\partial C=\varnothing$ we let $V=C$. If $\partial C \neq \varnothing$ then $\{x\}$ and $\partial C$ are disjoint nonempty closed subsets of $C$. Since $C$ is a normal space in the relative topology, we may apply Urysohn's lemma (see [6, p. 115]) to obtain a continuous function $\phi$ mapping $C$ into the unit interval and satisfying $\phi(x)=0$ and $\phi(y)=1$ for all $y \in \partial C$. Let $r$ be any number with $0<r<1$. Then $\phi^{-1}([0, r])$ is a closed subset of $C$ and hence a closed subset of $P$. Similarly $\phi^{-1}([0, r))$ is an open subset of $C-\partial C$ and hence an open subset of $P$. It follows that $\partial \phi^{-1}([0, r]) \subset \phi^{-1}([0, r])$ $-\phi^{-1}([0, r))=\phi^{-1}(r)$. We need only find an $r, 0<r<1$, with $\mu_{i}\left(\phi^{-1}(r)\right)$ $=0$ for each $i$. We may then set $V=\phi^{-1}([0, r])$. To see that there is such an $r$ we first note that $\left\{\phi^{-1}(r) \mid 0<r<1\right\}$ is a family of pairwise disjoint closed subsets of $C$. Now for a particular $i, \mu_{i}(C)$ is finite and hence there can only be a countable number of $r$ 's, $0<r<1$, with $\mu_{i}\left(\phi^{-1}(r)\right)>0$. Thus for all but a countable number of $r$ 's, $0<r<1$, we must have $\mu_{i}\left(\phi^{-1}(r)\right)=0$ for all $i$. The result follows.

The following is a type of measure-theoretic extension of Urysohn's lemma. The formulation we give is entirely adequate for our purposes though various generalizations are possible.

3.5. Lemma. If $P$ is compact and $\mu_{i}(\{x\})=0$ for each $i$ and each 
$x \in P$, then there is a continuous function $f$ mapping $P$ into the unit interval $I$ with $\mu_{i}\left(f^{-1}(r)\right)=0$ for each $i$ and each $r \in I$.

Proof. We begin with a preliminary construction. Let $i$ be a fixed positive integer and let $F \subset O$ where $F \in \mathcal{C}$ and $O \in Q$. By our hypotheses and Theorem $3.1, \mu_{i}$ has no atoms. Since $P$ is compact, $\mu_{i}(O-F)$ is finite and by Lemma 3.2 we can choose as et $B C O-F$ such that $\mu_{i}(B)=(1 / 2) \mu_{i}(O-F)$. By the regularity of $\mu_{i}$ there is a compact set $C \subset B$ with $\mu_{i}(C) \geqq(1 / 2) \mu_{i}(B)$. Again by regularity we can choose an open set $Q \subset O$ such that $\mu_{i}(Q-(F \cup C)) \leqq(1 / 4) \mu_{i}(O-F)$. By the normality of the space we may choose an open set $Q^{\prime}$ with $\left(Q^{\prime}\right)-C O$ and $(F \cup C) \subset Q^{\prime}$. Then again $\mu_{i}\left(Q \cap Q^{\prime}-(F \cup C)\right) \leqq(1 / 4) \mu_{i}(O-F)$. Now $P$ is closed under finite unions and is a base for the topology of $P$ by Lemmas 3.3 and 3.4 and since $F \cup C$ is compact there is an $R \in P$ with $F \cup C \subset R \subset Q \cap Q^{\prime}$. For this $R, F \subset R \subset R-\subset 0$,

$$
\begin{aligned}
\mu_{i}(R-F) & =\mu_{i}(R-(F \cup C))+\mu_{i}(C) \leqq(1 / 4) \mu_{i}(O-F)+\mu_{i}(B) \\
& \leqq(1 / 4) \mu_{i}(O-F)+(1 / 2) \mu_{i}(O-F)=(3 / 4) \mu_{i}(O-F),
\end{aligned}
$$

and

$$
\begin{aligned}
\mu_{i}(O-R) & \leqq \mu_{i}(O-(F \cup C))=\mu_{i}(O-F)-\mu_{i}(C) \leqq \mu_{i}(O-F)-(1 / 2) \mu_{i}(B) \\
& =\mu_{i}(O-F)-(1 / 4) \mu_{i}(O-F)=(3 / 4) \mu_{i}(O-F) .
\end{aligned}
$$

Using the axiom of choice we shall associate with each positive integer $i$ and pair of sets $F \in \mathcal{C}$ and $O \in Q$ with $F \subset O$, a definite $R$ obtained in the manner just described and denoted by $R(i, F, O)$.

As a second step let $\omega$ stand for the set of positive integers and let $g: \omega \rightarrow \omega \times \omega$ be a bijection. Then if $\pi: \omega \times \omega \rightarrow \omega$ is the projection onto the first coordinate and $h=\pi \circ g, h: \omega \rightarrow \omega$ and for each $n \in \omega, h^{-1}(n)$ is an infinite set. For each pair of integers $n$ and $i$ let $m(n, i)$ stand for the number of integers in $h^{-1}(i)$ which are less than or equal to $n$. Thus for each $i, \lim _{n} m(n, i)=\infty$.

We now employ the functions $R$ and $h$ to construct the function $f$. If $P$ is a singleton there is nothing to prove, hence, we shall suppose $P$ contains at least two distinct points $x_{0}$ and $x_{1}$. Let $D$ stand for the dyadic rationals in $[0, \infty)$. Set $F[t]=P$ for $t \in D, t>1, F[1]=P-\left\{x_{1}\right\}$, and $F[0]=\left\{x_{0}\right\}$. In general for $0<t<1$ with $t \in D$ we can write $t$ $=(2 m+1) 2^{-n}$ and this representation is unique. We complete the definition of the $F[t]$ 's, $t \in D$, by recursion on $n$. If $n=1$ we must have $t=1 / 2$ and we set $F[1 / 2]=R\left(h(1),(F[0])^{-}, F[1]\right)$. For larger $n$ we set

$$
F\left[(2 m+1) 2^{-n}\right]=R\left(h(n),\left(F\left[m 2^{-(n-1)}\right]\right)-, F\left[(m+1) 2^{-(n-1)}\right]\right) .
$$

The function $f$ is then defined by 


$$
f(x)=\inf \{t \in D \mid x \in F[t]\}
$$

for all $x \in P$. The continuity of $f$ follows in the usual way (see [6, p. $114])$. We proceed to prove by induction on $n$ that

$$
\mu_{i}\left(F\left[(2 m+1) 2^{-n}\right]-F\left[2 m 2^{-n}\right]\right) \leqq(3 / 4)^{m(n, i)} \mu_{i}(P),
$$

and

$$
\mu_{i}\left(F\left[(2 m+2) 2^{-n}\right]-F\left[(2 m+1) 2^{-n}\right]\right) \leqq(3 / 4)^{m(n, i)} \mu_{i}(P) .
$$

The inequalities plainly hold when $n=1$. Assume them for $n-1 \geqq 1$. Then

$$
\begin{aligned}
\mu_{i}(F[(2 m & \left.+1) 2^{-n}\right]-F\left[2 m 2^{-n}\right]=\mu_{i}\left(F\left[(2 m+1) 2^{-n}\right]-\left(F\left[2 m 2^{-n}\right]\right)^{-}\right) \\
& \leqq(3 / 4)^{(m(n, i)-m(n-1, i))} \mu_{i}\left(F\left[(m+1) 2^{-(n-1)}\right]-F\left[m 2^{-(n-1)}\right]\right) \\
& \leqq(3 / 4)^{m(n, i)} \mu_{i}(P)
\end{aligned}
$$

and similarly $\mu_{i}\left(F\left[(2 m+2) 2^{-n}\right]-F\left[(2 m+1) 2^{-n}\right]\right) \leqq(3 / 4)^{m(n, i)} \mu_{i}(P)$. For $r \in I, f^{-1}(r)=\bigcap_{t>r} F[t]-\bigcup_{t<r} F[t]$ and it is not difficult to see that $\mu_{i}\left(f^{-1}(r)\right) \leqq 2(3 / 4)^{m(n, i)} \mu_{i}(P)$ for each $n$. Thus $\mu_{i}\left(f^{-1}(r)\right)=0$ for each $i$.

Important for our construction of nonmeasurable sets is the following lemma which appears as an exercise in $[5$, p. 146] and is there credited to $\mathrm{F}$. Bernstein. We leave the proof to the reader.

3.6. Lemma. Let I be the unit interval with the usual topology. Then there is a subset $S$ of I such that the cardinal of any closed subset of $S$ or $I-S$ is at most $\boldsymbol{\aleph}_{0}$.

If $(X, \mathfrak{T}, \mu)$ is a measure space and $Y \subset X$ we shall let

$$
\mu^{*}(Y)=\inf \{\mu(A) \mid A \in \mathfrak{N}, Y \subset A\}
$$

and

$$
\mu_{*}(Y)=\sup \{\mu(A) \mid A \in \mathfrak{T}, A \subset Y\} .
$$

The numbers $\mu^{*}(Y)$ and $\mu_{*}(Y)$ are called the outer and inner measure of $Y$, respectively (see [3, p. 58]).

3.7. Theorem. Let $\left\{\left(P, \mathfrak{T}_{i}, \mu_{i}\right)\right\}_{i=1}^{\infty}$ be a family of measure spaces with each $\mu_{i}$ regular. Further suppose $\mu_{i}(P)>0$ for each $i$ and $\mu_{i}(\{x\})=0$ for each $i$ and each $x \in \dot{P}$. Then there is a subset $T$ of $P$ such that $T$ $\notin \cup_{i=1}^{\infty} \mathfrak{T}_{i}$. If $P$ is compact $T$ may be so chosen that $\mu_{i}^{*}(T)=\mu_{i}(P)$ and $\mu_{i_{*}}(T)=0$ for each $i$.

Proof. We begin by considering the case when $P$ is compact. Let $S$ be a subset of $I$ with the properties described in Lemma 3.6 and let $f: P \rightarrow I$ be as in Lemma 3.5. Set $T=f^{-1}(S)$. Then $P-T=f^{-1}(I-S)$. 
It will be sufficient to show that $\mu_{i_{*}}(T)=\mu_{i_{*}}(P-T)=0$ for each $i$. By symmetry we need only show $\mu_{i_{*}}(T)=0$ for each $i$. Suppose $\mu_{i_{*}}(T)>0$ for some $i$. Then by the regularity of $\mu_{i}$ there must be a compact set $C$ with $C \subset T$ and $\mu_{i}(C)>0$. Since $\mu_{i}\left(f^{-1}(r)\right)=0$ for each $r \in I$, we must have $C \cap f^{-1}(r) \neq \varnothing$ for an uncountable number of $r \in I$. Thus $f(C)$ is an uncountable closed subset of $S$, which is a contradiction.

There remains the case when $P$ is not compact. We choose a sequence of compact sets from $P$ in the following manner. Let $C_{1}$ be a compact set with $\mu_{1}\left(C_{1}\right)>0$. Suppose all $C_{i}$ 's up to $C_{n}$ have been chosen. Then if $\mu_{n+1}\left(\cup_{i=1}^{n} C_{i}\right)=0$ let $C_{n+1}$ be a compact set with $C_{n+1}$ $\bigcap\left(\cup_{i=1}^{n} C_{i}\right)=\varnothing$ and $\mu_{n+1}\left(C_{n+1}\right)>0$. Otherwise let $C_{n+1}=\varnothing$. We next choose a corresponding sequence $\left\{T_{n}\right\}_{n=1}^{\infty}$. If $C_{n}=\varnothing$ let $T_{n}=\varnothing$. If $C_{n} \neq \varnothing$ let $T_{n}$ be a subset of $C_{n}$ which is not measurable by any $\mu_{i}$ with $\mu_{i}\left(C_{n}\right)>0$. Let $T=\bigcup_{n=1}^{\infty} T_{n}$. If $T$ were measurable by $\mu_{i}$ so would $T_{n}=T \cap C_{n}$ be for each $n$, which is not the case. Thus $T \in \bigcup_{i=1}^{\infty} \mathfrak{T l}_{i}$.

\section{REFERENCES}

1. P. J. Cohen, Set theory and the continuum hypothesis, Benjamin, New York, 1966.

2. J. Dugundji, Topology, Allyn and Bacon, Boston, Mass., 1966.

3. P. R. Halmos, Measure theory, Van Nostrand, New York, 1950.

4. E. Hewitt and K. A. Ross, Abstract harmonic analysis. I, Springer-Verlag, Berlin, 1963.

5. E. Hewitt and K. Stromberg, Real and abstract analysis, Springer-Verlag, New York, 1965.

6. J. L. Kelly, General topology, Van Nostrand, New York, 1955.

7. J. R. Shoenfield, Mathematical logic, Addison-Wesley, Reading, Mass., 1967.

Pacific Union College 